\title{
Germanica
}

$47 \mid 2010$

« Krack! Tschock ! Pflatsch ! Bummmm ! La BD de langue allemande (à suivre...) »

\section{Gerhard Haderer, un regard acéré sur la vie en Autriche}

Gerhard Haderer, a scathing sketch of life in Austria.

\section{Anne-Marie Corbin}

\section{OpenEdition}

\section{Journals}

Édition électronique

URL : http://journals.openedition.org/germanica/1126

DOI : $10.4000 /$ germanica. 1126

ISSN : 2107-0784

Éditeur

Université de Lille

Édition imprimée

Date de publication : 31 décembre 2010

Pagination : 99-125

ISBN : 9782913857261

ISSN : 0984-2632

Référence électronique

Anne-Marie Corbin, « Gerhard Haderer, un regard acéré sur la vie en Autriche », Germanica [En ligne], 47 | 2010, document 8, mis en ligne le 01 décembre 2012, consulté le 06 octobre 2020. URL : http:// journals.openedition.org/germanica/1126 ; DOI : https://doi.org/10.4000/germanica.1126

Ce document a été généré automatiquement le 6 octobre 2020.

(c) Tous droits réservés 


\title{
Gerhard Haderer, un regard acéré sur la vie en Autriche
}

Gerhard Haderer, a scathing sketch of life in Austria.

\author{
Anne-Marie Corbin
}

1 Dans le film d'Ulrich Seidl, tourné en 1991, Gerhard Haderer ${ }^{1}$ affirme : « Mes dessins sont des histoires en images. Je suis un narrateur. Il s'agit pour moi de raconter le mieux possible une histoire ${ }^{2}$ ». Ces histoires en images prennent diverses formes, depuis le dessin ou la planche dans des hebdomadaires jusqu'à des albums où l'histoire unique se déroule avec ses péripéties tout au long du volume autour d'un ou de plusieurs héros.

2 Né en 1951 à Leonding près de Linz, Haderer a fait des études de graphisme publicitaire, complétées par un apprentissage de gravure à Stockholm. Après un premier emploi de décorateur pour Quelle, une entreprise allemande de vente par correspondance, il se met à son compte et travaille pour des agences de publicité. En 1984, renonçant à ces activités qu'il considère comme trop mercantiles, il décide de sauter le pas pour se consacrer au dessin satirique, qui lui tient vraiment à cœur. Il fait ses débuts dans la presse régionale, Oberndorf aktuell, et une revue satirique, Watzmann ${ }^{3}$. Bientôt, il dispose d'une page hebdomadaire ou bimensuelle dans Profil et Stern. On lui consacre peu à peu des expositions, ses travaux sont rassemblés dans des livres et il se met, depuis les années 1990, à créer des albums originaux. Son évolution la plus récente : en mai 2010, il se lance avec ses «Fliegende Blätter» («Feuilles volantes») dans une campagne de soutien au SPÖ et à Häupl pour l'élection du maire de Vienne.

Sa production s'étend donc sur plus d'une vingtaine d'années.

\section{Attention, il vous regarde !}

4 Gerhard Haderer a travaillé pour une agence de publicitét. Il y a acquis une technique, mise au service de l'efficacité. Il y a appris comment toucher son public, le séduire ou le surprendre en se donnant les moyens d'y parvenir. Pour dessiner régulièrement dans 
des hebdomadaires, il faut aussi avancer vite, ce qui ne signifie pas pour autant bâcler son ouvrage, puisque l'élaboration d'un dessin en couleur - toujours techniquement très abouti - lui prend en moyenne une vingtaine $d^{\prime}$ heures ${ }^{5}$. Cela implique tout un travail préparatoire en amont.

Son sujet de prédilection étant les travers de la vie quotidienne en Autriche, Haderer se promène, un sac en bandoulière, dont il sort son précieux carnet de croquis. Tantôt, il dessine au café deux dames d'un certain âge qui dégustent leurs gâteaux d'un air gourmand. Tantôt, il se rend à la gare et y saisit l'expression d'un homme qui boit sa bière à grands traits, en attendant son train de banlieue, le visage fatigué par sa journée de travail. On peut aussi le rencontrer dans un club de gymnastique où il croque une jeune femme en train de s'exercer aux machines ${ }^{6}$ ou un athlète qui pratique le bodybuilding ${ }^{7}$. C'est l'Autriche de tous les jours, celle des gens qu'il côtoie, et l'on reconnaît parfois l'expression d'une voisine sur un dessin. Dans son carnet, il multiplie les esquisses, les conserve pour pouvoir, un jour, en sélectionner un fragment et, l'ajoutant à d'autres, trouver ce dont il aura besoin. Sur la route des vacances, il croque férocement les touristes - allemands également - avec leurs travers, jusque sur le lieu de leur villégiature ${ }^{8}$. Il ne cesse pas non plus de dessiner le soir en regardant la télévision : il étudie alors les hommes politiques en action et consigne les événements qui feront peut-être l'objet de son dessin de la semaine dans des hebdomadaires comme Stern ou Profil, pour lesquels il a travaillé jusqu'à une époque récente ${ }^{9}$. Haderer observe, son regard est acéré et ne laisse rien passer.

6 Ces personnages de la vie de tous les jours, il les place dans le décor qui leur est familier et que l'on reconnaît immanquablement à de nombreux détails. Ce sont les petits galets bien polis qui pavent les places et les rues des villes et villages autrichiens. Ce sont les maisons typiques aux couleurs clinquantes si identifiables. Pour les intérieurs aussi, il lui faut coller à la réalité. Pour ce faire, sa collaboration avec la maison Quelle lui a été fort utile. Il a pris l'habitude de collectionner des catalogues où il trouve le modèle exact des objets de la vie quotidienne, des chaussures ou des vêtements à la mode du moment jusqu'aux meubles qu'apprécie la petite bourgeoisie mise en scène dans ses dessins. Mais la critique implicite pointe dans son choix : si l'un de ses personnages est assis sur un canapé, il le choisit recouvert d'un imprimé hideux, il va insister sur les accoudoirs disproportionnés jusqu'à la monstruosité et bien montrer l'alignement ridicule des coussins et le napperon de protection ${ }^{10}$. L'observation se transforme petit à petit en une critique de la société de consommation qu'il veut épingler en la représentant dans des scènes bien réelles.

7 Puis, à partir de quelques croquis, une idée va germer et se concrétiser peu à peu. Comme Haderer nous le confirme dans l'entretien téléphonique que nous avons réalisé le 2 juin 2010, sa technique s'est perfectionnée au fil des ans, mais elle reste inchangée, car elle est efficace.

8 Le travail est très minutieux et a pour base une esquisse au crayon. Le dessin prend souvent forme en petit format avant d'être projeté sur un carton à la dimension désirée à l'aide d'un épiscope ${ }^{11}$. Haderer reprend alors ses crayons, ses feutres et sa plume et suit les contours à main levée, parfois aussi à l'aide d'un pinceau très fin. Pour les traits du visage, il utilise un feutre ou des crayons de différentes tailles et étudie ses propres mimiques dans un miroir grossissant. Il découpe certains éléments au cutter, en particulier pour détourer. À l'aide d'un calque, il va intégrer le décor et les éléments qu'il emprunte à des catalogues, d'autres objets comme son chapeau fétiche étant posés 
sur sa table de travail. C'est le moment où la couleur intervient, grossièrement d'abord avec une peinture acrylique, aussi fluide que l'aquarelle, au pinceau épais pour le fond, qu'il va gratter par endroits en superposant des couches de différentes couleurs. Il les pulvérise plus rarement à l'aérographe. Les finitions se font avec des feutres très fins et des crayons de couleur. À partir du travail de préparation, évoqué ci-dessus, Haderer peut même battre son record de rapidité en finalisant un dessin en deux bonnes journées de travail. L'un d'eux, celui qu'il présente dans la postface de Think positive pour illustrer sa méthode de travail, va trouver sa place face à un article dans un magazine économique traitant des salaires en Autriche : son " mendiant dans le luxe", assis contre un mur de briques, éclairé par un spot qui met bien en évidence le contraste avec les montres rollex, les chaussures italiennes et le fameux chapeau à ses pieds, et surtout la dentition parfaite, résultat d'un onéreux traitement chez un dentiste ${ }^{12}$. Le message de Haderer est clair : certes, comme ce mendiant, les Autrichiens réclament toujours plus à leurs hommes politiques, mais leurs signes extérieurs de richesse révéleraient qu'ils ne sont pas à plaindre.

\section{Le refus de l'hypocrisie (une chronique de la vie quotidienne)}

9 Les dessins de Haderer effectuent une remise en cause radicale des clichés sur la « belle Autriche", en usage en particulier dans les guides touristiques: "En tant que caricaturiste, j'ai prôné pendant des décennies la vigilance politique en tentant de dépasser ses contingences régionales et continuerai à le faire ${ }^{13}$ ».

Le lecteur se souviendra peut-être d'une exposition du Österreichisches Museum für Volkskunde de 1996 et de son catalogue ${ }^{14}$. Il s'agissait, d'une autre manière que chez Haderer, de conceptualiser les notions de Heimat (" petite patrie ») et de Heimatschutz (" protection de la petite patrie ») et d'en montrer les connotations idéologiques dans la tradition autrichienne, notamment la marque imprimée par le nationalisme et les relents de national-socialisme. La démarche de Haderer ne peut se comprendre sans la référence à ces réflexions qui, en Autriche en particulier, déterminent les prises de parti et les attitudes de tout un chacun.

11 Il ne faut pas oublier qu'au plus tard après la dissolution de l'Empire austro-hongrois à la fin de la Première Guerre mondiale, le territoire actuel de l'Autriche devient - selon le mot de Clemenceau - "ce qui reste», c'est-à-dire qu'il est réduit à la portion congrue. Cette situation imposée lors du Traité de Saint-Germain par les Alliés victorieux fut ressentie très fortement comme une humiliation et exacerba tous les nationalismes, sécrétant également la peur d'un Etat non viable, ainsi que d'une perte d'identité. L'annexion volontaire à l'Allemagne fut interdite en dépit des aspirations existant à la fois des côtés allemand et autrichien. Avant même "l'Anschluss » de 1938, la démocratie parlementaire fut démantelée le $1^{\mathrm{er}}$ mai 1934 avec l'arrivée au pouvoir de l'austro-fascisme et de son "Front patriotique " (Vaterländische Front), appelant à un rassemblement de toutes les couches de la population autour d'une idéologie passéiste proclamant une «nouvelle Autriche patriotique ». On assista à un retour en arrière, à la référence à des clichés et des stéréotypes ${ }^{15}$ qui figeaient la société dans l'idéal d'une nature intacte et d'une paysannerie garantissant la pérennité d'un monde soi-disant harmonieux, ennemi de la modernité et de l'esprit des métropoles. 

voire réactionnaires de la société autrichienne (ÖVP et FPÖ). Haderer montre résolument l'autre face de la réalité. Les Alpes, symbole même d'une nature vierge, sont devenues des montagnes dévastées par la pratique du ski de piste, et des files de skieurs à la mode les dénaturent chaque jour davantage ("Schno», in Think positive). Le tourisme à échelle industrielle a bien contribué au désastre écologique qu'il constate : «Pizzeria Hoamatland» (1988, in Think positive) montre un couple d'une cinquantaine d'années devant l'hôtel à sept étages qu'il a construit au milieu des montagnes. La photo que la femme exhibe avec fierté indique que le paysage était encore intact une vingtaine d'années auparavant. Chamois et edelweiss, autres symboles de cette nature autrichienne, y subsistaient peut-être encore. " Hoamatland », "Heimat » en dialecte autrichien: ainsi se nomme le fameux hôtel, ce qui permet à Haderer de dénoncer l'implantation dans ce panorama d'un tel immeuble, véritable caricature en béton de la ferme traditionnelle. En outre, le port du costume traditionnel - les vêtements en loden et peut-être le pantalon de cuir pour l'homme - est aussi une des images idéales significatives d'une mise en scène de la province autrichienne, destinée à la fois à mobiliser les traditionalistes et à appâter le touriste.

Si Haderer est aussi virulent contre cette province autrichienne, c'est également parce qu'elle exclut ceux qui n'en sont pas originaires. Les signes d'appartenance vont de pair avec des mots d'ordre de rejet de l'étranger, l'image de soi valorisante de l'Autriche signifiant la dévalorisation et le rejet de tout ce qui n'en fait pas partie. "Asylanten raus " (1991, in Vorsicht Haderer) montre une crèche vide où les murs de l'étable sont couverts d'inscriptions malveillantes et assassines : «Les demandeurs d'asile dehors». Les images de skinheads, l'un d'eux se réjouissant d'avoir passé des vacances à Rostock, un autre couvrant les murs de croix gammées, sont fréquentes dans ses dessins et ses albums. C'est dire que Haderer fait le lien entre le présent et le passé nazi de l'Autriche pour reprocher à certains de ses compatriotes de persister dans leurs dénis et leur refoulement du rôle qu'ils ont joué à l'époque du Troisième Reich (« Der Beweis : viele haben wirklich nicht gewusst ", in Think positive). Ainsi, Haderer s'éleva fermement contre la présence de Kurt Waldheim à la tête de l'État autrichien et les trop vagues regrets exprimés à la date anniversaire de la « Nuit de cristal » de novembre 1938 pour le pogrom contre les Juifs (1988, " Wir bedauern... », in Think positive).

Dans Von Hunderl und Menschen, album publié en $2003^{16}$, la province autrichienne est à nouveau thématisée avec une présentation différente. Une double page rassemble des personnages autour des tables de la terrasse d'un café, suivie d'une autre double page, fonctionnant avec des vignettes sur le mode des planches de bandes dessinées et développant un aspect particulier de la vie d'un des personnages. Les épisodes s'enchaînent pour constituer une chronique de la ville où défilent Gusenbauer, George W. Bush en chanteur de country-music, Karl Habsbourg (le fils du dernier empereur autrichien), Pierre Brice déguisé en Winnetou, Patrick Ortlieb et aussi le chancelier Schüssel, la seule de ces séquences que nous présenterons ici. Schüssel, en costumecravate gris, est coiffé d'un grand chapeau auquel le ruban confère un caractère folklorique et lui donne l'allure d'un gnome. Il est tourné en ridicule, car il s'impose à la table de deux femmes d'une quarantaine d'années en annonçant qu'il serait « l'une des personnalités les plus fascinantes de ce pays ». Sûr d'être reconnu, il ne se présente pas. Image après image, on le voit fort dépité, son rictus s'accentuant et l'étonnement se dessinant sur son visage. Selon Haderer, Schüssel n'a jamais été qu'un personnage de 
second plan, totalement dépourvu d'aura. Il n'a dû sa carrière qu'aux hasards de la vie politique, mais nullement à ses qualités personnelles.

À ces critiques de la vie en Autriche s'est ajoutée plus récemment une thématique plus globale, moins centrée sur l'Autriche, celle de la remise en cause des symboles de la modernité virtuelle (ordinateurs, portables, etc.). Certes, Haderer a toujours abhorré les chasseurs. Mais, dans un dessin destiné à Stern,de bourreaux, ils deviennent victimes ${ }^{17}$. Au milieu d'une belle forêt de pins, on voit un homme étendu par terre, son portable à ses côtés. Un autre se couvre les yeux d'un air désespéré, car il vient de tirer sur son compagnon. Le texte permet de comprendre que le chasseur avait choisi pour sonnerie le brame du cerf. Avec « Der Bücherwurm » («Le rat de bibliothèque », Stern, $\left.2009, \mathrm{n}^{\circ} 32\right)$, les nouvelles techniques ont vidé les rayonnages des bibliothèques traditionnelles. La situation frise l'absurde, car l'homme fort âgé - à la chevelure très blanche - que l'on voit sur le dessin persiste à grimper sur son escabeau dans un lieu qu'il affectionne, mais qui a perdu tout intérêt puisqu'il suffit dorénavant de consulter ses livres sur un Ipad, l'un de ces «e-readers» qui proposent de télécharger des millions de livres.

Enfin, on perçoit une réflexion sur le temps qui passe, toute la génération de Haderer basculant dans la vieillesse. Dans «Beatles Revival», un vieux chanteur de rock entonne Yellow Submarine devant une assemblée de retraités, le club 1968 (in Think positive). Avec «Beatles 1996 », des Beatles vieillis dont le groupe est réduit à trois après la disparition de John interviennent dans Think positive. Cette même thématique est encore déclinée dans Stern avec «Die Fans werden älter - der Konflikt bleibt » («Les fans vieillissent, le conflit demeure ", in Stern, 2007, n 29)et "Woodstock - 40 Jahre später » («Woodstock, quarante ans après », in Stern, 2009, n 35 ) :les attitudes et les conflits n'ont guère changé, mais les protagonistes ont une quarantaine d'années de plus.

\section{Le profond rejet de l'Église catholique et de ses dogmes}

17 Haderer s'attaque inlassablement aux puissants de ce monde pour dénoncer leurs agissements :

La seule chose qui l'intéresse chez les gens connus est de dévoiler le système qu'ils représentent et d'en illustrer le fonctionnement à l'aide d'instantanés en couleurs vives que l'on peut, à sa guise, appeler des hasards, des accidents ou des cas particuliers ${ }^{18}$.

En premier lieu, il prend fréquemment pour cible les ecclésiastiques, représentants d'une Église catholique très puissante en Autriche, où il n'y a pas de séparation de l'Église et de l'État. Ses dessins deviennent alors de véritables caricatures pour dénoncer les outrances idéologiques d'une institution. Dans «Priester» (1989, in Vorsicht Haderer, p. 50), il montre quatre prêtres et officiels de l'Église autrichienne, avançant l'un derrière l'autre d'un air belliqueux. Comme à l'époque de l'Inquisition ou celle, plus récente, où l'index établissait la censure, chacun d'eux brandit un épais volume au titre programmatique : l'un dénonce le sida comme étant la « juste punition divine ", l'autre la démocratie comme "l'œuvre du diable », un troisième assimile la liberté à un fléau, le dernier dénonce les pratiques homosexuelles. C'est l'évêque Kurt Krenn qui mène la ronde, représenté encore en 1993 (in Vorsicht Haderer, p. 25) nu, en 
prière devant un crucifix et un Christ filiforme, son obésité n'en étant que plus flagrante. Outre l'insistance sur le décalage entre la théorie d'abstinence, de tempérance et la pratique, c'est aussi un moyen indirect de rappeler que les prêtres ont un corps, et ce dès avant les scandales récents soulevés par les révélations sur l'homosexualité et la pédophilie de certains d'entre eux. A contrario, un prêtre sans nez (in Vorsicht Haderer, p. 52, "Johannes " $1992^{19}$ ) soulève sa soutane et dévoile son basventre asexué. Haderer s'en prend, ainsi, aux déclarations de l'Église qui refuse de prendre en compte la sexualité, tout en la pratiquant en cachette de manière hypocrite et en poursuivant son rôle de donneuse de leçons et de thuriféraire d'une morale répressive.

C'est avec La Vie de Jésus (Das Leben des Jesus) ${ }^{20}$ que Haderer va causer un véritable scandale public. L'album se vend en Autriche à 100000 exemplaires, est immédiatement traduit en français (seulement une centaine d'exemplaires en sont vendus !). Il y met en scène un Jésus assez sympathique, un peu hippy, l'air plutôt absent, car drogué à l'encens, et qui finit par trouver refuge au ciel $^{21}$ pour échapper à ses disciples corrompus par le pouvoir.

21 Haderer insiste sur l'enfance et la jeunesse de Jésus, c'est-à-dire la partie de sa vie sur laquelle les Évangiles gardent le silence. La lumière divine qui entoure Jésus sous forme d'auréole permet à Joseph de travailler de nuit et éclaire son entreprise florissante de menuiserie (anachronisme amusant des modèles de chaises qu'il fabrique en série, tel un précurseur des frères Thonet). S'agit-il déjà de blasphème? Cette lumière éblouissante attire les enfants dont la petite bande grandit peu à peu pour atteindre le nombre de douze ${ }^{22}$, car ils peuvent jouer de nuit à la marelle (en allemand « Himmel und Erde », c'est-à-dire " ciel et terre »). On reconnaît encore parmi eux la figure du chancelier autrichien ÖVP Wolfgang Schüssel. À l'instar des ecclésiastiques que campe Haderer, les disciples prennent de l'embonpoint, car ils profitent des soi-disant miracles de Jésus pour encaisser les récompenses en espèces sonnantes et trébuchantes, se faire bâtir des palais et mener grande vie. Affalés dans leur graisse, ils sont incapables de se mouvoir et se font transporter en chaise à porteurs. Une telle dénonciation des abus de pouvoir entre alors dans le domaine de la politique.

Mais Haderer tourne également le dogme en dérision de manière parodique. Les miracles sont devenus sous ses crayons un effet du hasard : quand Jésus marche sur les eaux, il utilise une sorte de planche à voile qui avance poussée par un vent favorable. C'est là l'image qui a le plus irrité l'archevêque Christoph Schönborn ${ }^{23}$ quand il s'en est pris à Haderer, davantage encore que la passion du Christ, représentée comme un autre hasard, Jésus fatigué, s'allongeant sous un arbre pour s'y reposer les bras en croix. L'image classique de la Cène - parodiant le célèbre tableau de Léonard de Vinci - est aussi reprise, avec Jésus au centre des apôtres (ivres et paillards), mais apparaissant ici dans le nuage d'encens qui le grise, tandis qu'il transforme l'eau en vin avec pour résultat une scène de beuverie digne de tableaux flamands. Symbole de l'opulence et du pouvoir, le futur clergé se pare de la tiare ou d'autres coiffures, de forme conique, très fantaisistes où les ors brillent à la lumière.

Haderer insiste sur la commercialisation du crucifix et des objets pieux dans une atmosphère de foire, un moyen pour les disciples de s'enrichir davantage, et qui répugne profondément à Jésus. Comme dans le film La Vie de Brian des Monty Python, l'accent est mis à la fois sur la corruption des intéressés et sur la bêtise des suiveurs. Le burlesque naît du décalage avec la représentation traditionnelle. 
Chacune des doubles pages de l'album, des images planches, constitue une fresque. Les interactions sont proches du cinéma muet, avec un texte sur le côté ou sous le dessin, parodiant les Évangiles par son style pour renforcer le message. Les énoncés se combinent pour former une totalité cohérente et en rendre l'impact plus virulent.

5 L'effet de provocation consistant à vouloir « raconter la vraie vie de Jésus et de ses disciples » ne reste pas sans effets en 2002. Sur un mode humoristique encore, l'éditeur Ueberreuter lance un forum de discussion sur le thème «A-t-on le droit de faire des plaisanteries sur Jésus? » et obtient un an encore après la parution de l'album quelque cinq cents réponses. Il propose également toute une liste de liens pour se documenter sur Dieu, la Bible, le Vatican, etc. et ajoute :

Nous renvoyons toute personne voulant vérifier les longues recherches historiques, l'étude pénible des textes originaux, les onéreux voyages de recherche et les innombrables analyses d'encens, entreprises par Gerhard Haderer, aux diverses éditions de la Bible, par exemple... ${ }^{24}$

Si Haderer reçoit en Autriche des menaces de mort à la suite de son album iconoclaste, le comble de la réaction est atteint en Grèce avec sa condamnation par contumace à six mois de prison. À la campagne contre lui lancée par l'archevêque Schönborn, Haderer réplique en se référant à l'article 188 du code pénal autrichien qui punit pour blasphème à la fois « le dénigrement des religions et celui des représentants de Dieu sur terre » :

28 Cet article doit être rayé immédiatement dans son intégralité. C'est un reliquat de l'obscurantisme du Moyen Âge. De plus, la liberté de l'art existe, souvent toutefois en contradiction avec tout cela. Il y a ici une incompatibilité totale entre l'Aufklärung des 250 dernières années et les dogmes de l'Église. C'est ce que l'on appelle un clash des civilisations. J'ai l'impression qu'on assiste en ce moment à un retour diffus de la religiosité, sa forme la pire en étant le slogan politique «L'Occident entre les mains du Christ ${ }^{25}$.

Ainsi, Haderer inscrit sa provocation blasphématoire dans la lignée de l'Aufklärung, les Lumières des pays de langue allemande, partant en campagne contre les mensonges et l'intolérance des religions, ainsi que des religieux et de leurs liens avec le pouvoir politique : «Le manque de respect est la base même de la caricature et de l'expression satirique sous quelque forme que ce soit ${ }^{26} »$. 


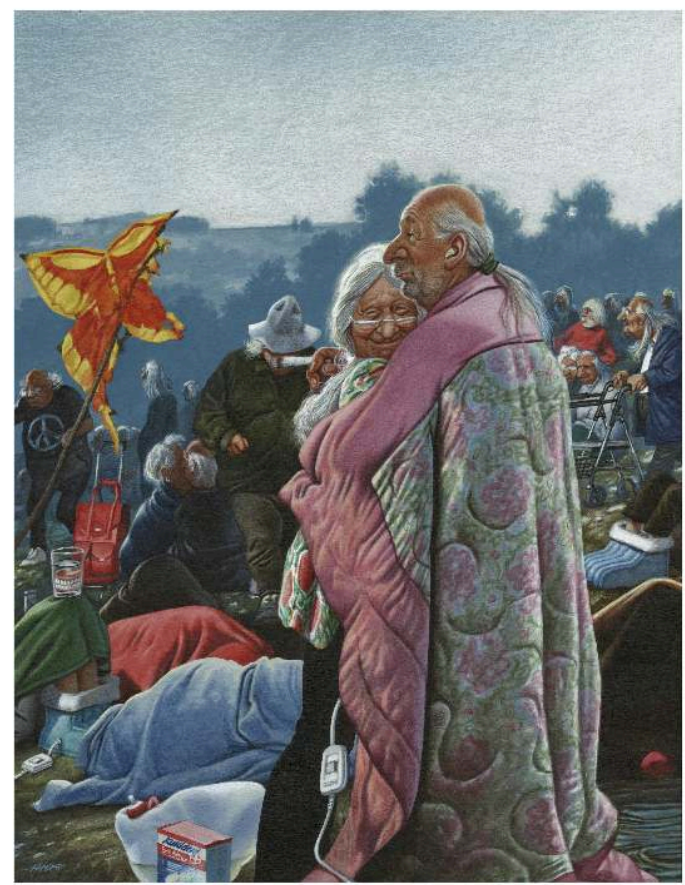

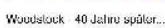




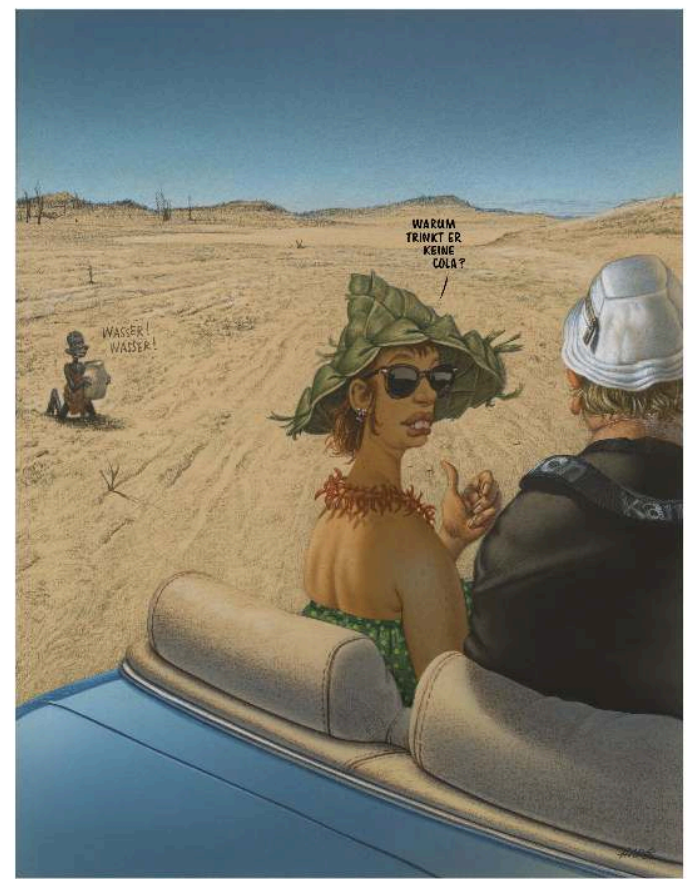

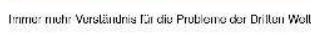

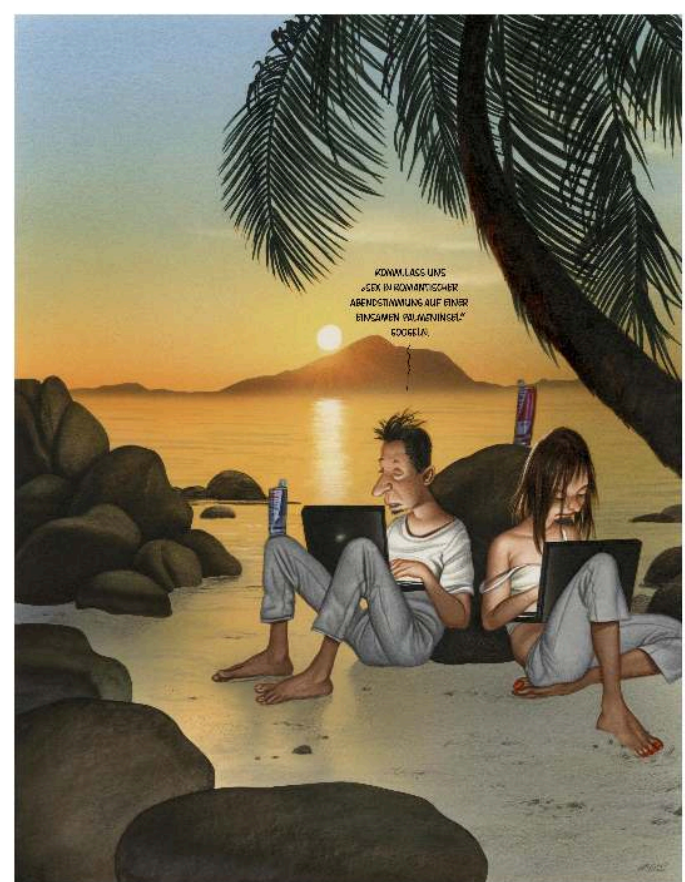



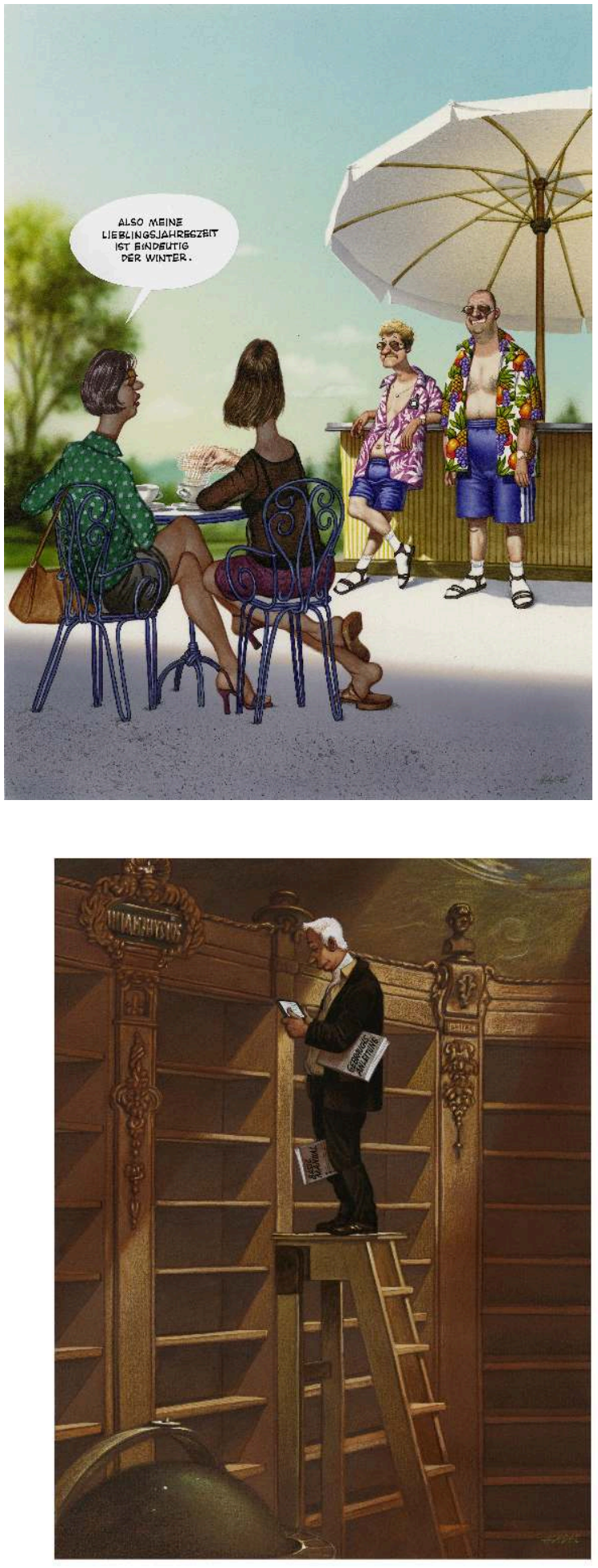

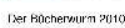




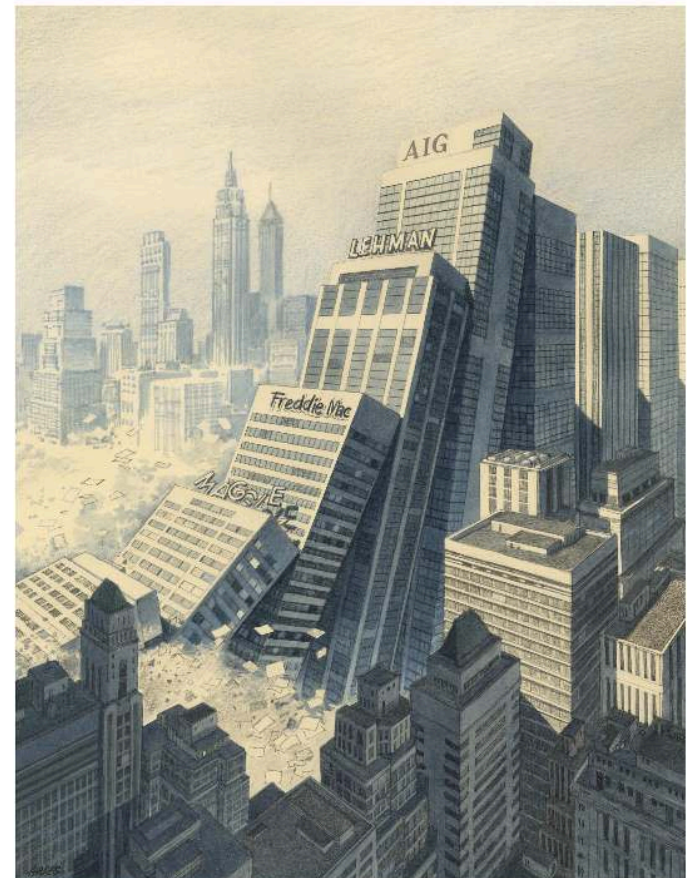

wallarreet - Doromin

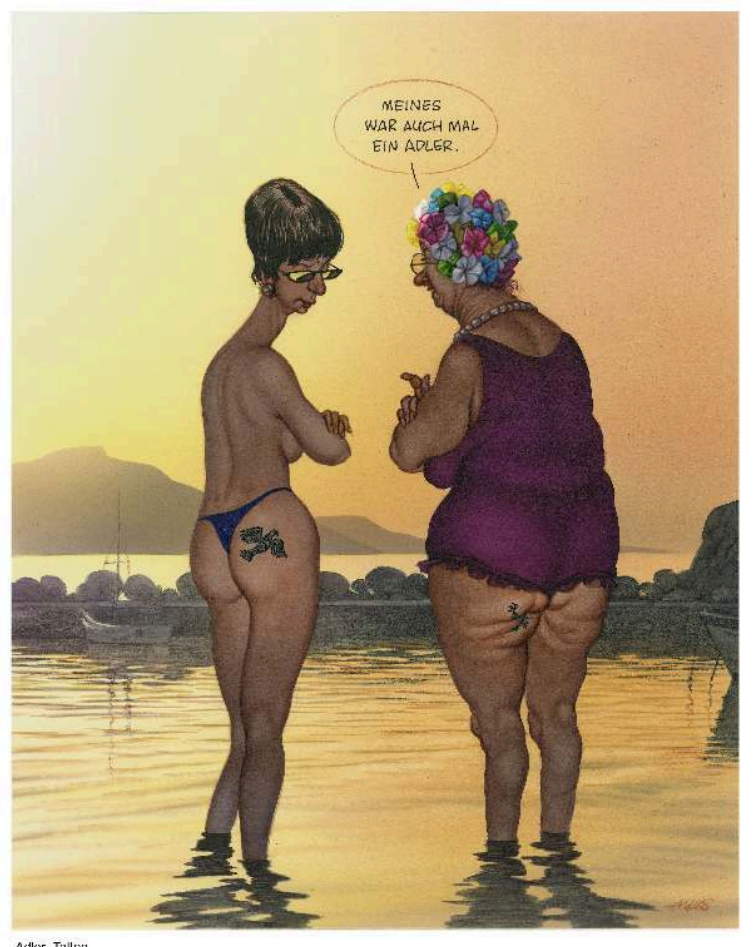



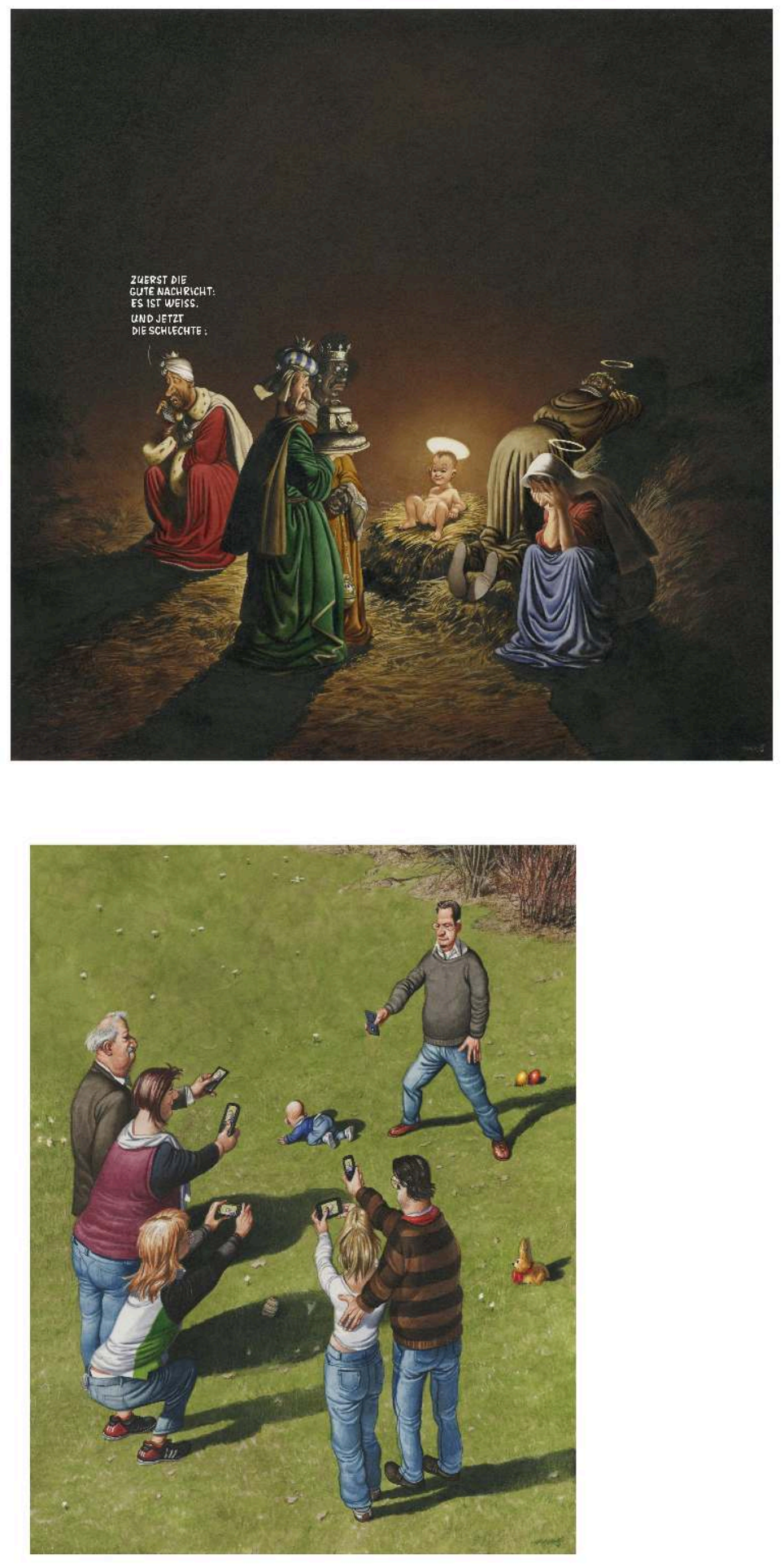

Furto ostar, 

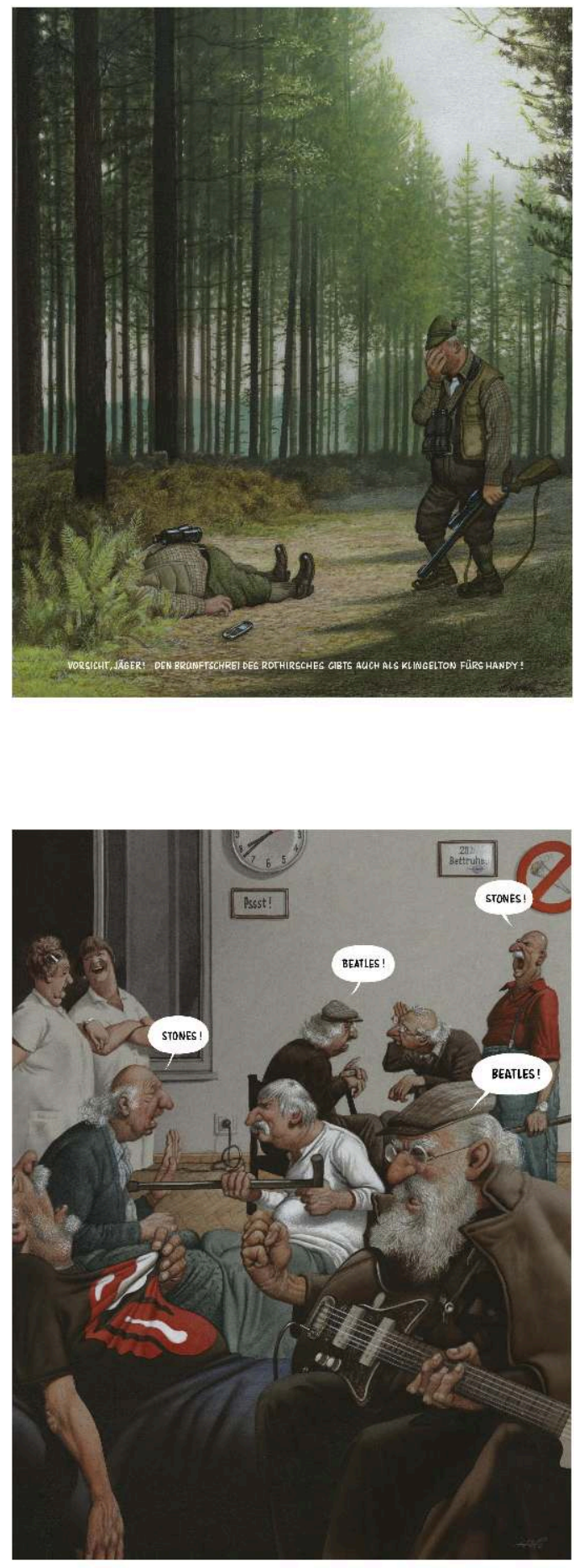

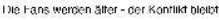

\section{Des hommes politiques mis au pilori}

Les caricatures d'hommes politiques de tout bord sont fréquentes dans les dessins de Haderer. Puisqu'il dessine dans Stern, il s'en prend souvent au chancelier Helmut Kohl, représenté en 1993 marchant sur les eaux, en sandales et chaussettes - selon le cliché 
de l'Allemand en vacances -, sous le regard interloqué de deux cygnes ${ }^{27}$. En revanche, Haderer ménage davantage Angela Merkel, qu'il a dessinée sous les traits d'un ange bleu au visage quelque peu fatigué (Stern, 23 mars 2000) et considère comme l'antithèse de Kohl, bien qu'ils appartiennent au même parti politique, la $\mathrm{CDU}^{28}$.

Si Haderer n'est pas un homme de parti, s'il n'entretient guère de liens avec les Verts, il faut reconnaître qu'il prend le plus souvent pour cible la droite conservatrice et l'extrême droite autrichiennes. C'est d'ailleurs l'objet d'un autre album que nous voulons présenter, Jörgi, der Drachentöter (" Jörgi, le tueur de dragons ») ${ }^{29}$. L'action se déroule au Moyen Âge, dans un décor de carton-pâte, où des détails anachroniques (certains panneaux et costumes, les jouets des enfants) rappellent que nous vivons au $\mathrm{XXI}^{\mathrm{e}}$ siècle et amusent par ce décalage. Gerhard Haderer, en collaboration avec Leo Lukas pour le texte, campe le personnage du petit Jörgi (un diminutif presque affectueux de Jörg Haider, en 2000 chef du FPÖ, le parti populiste autrichien), un petit fanfaron, un chaudron sur la tête en guise de heaume, une lance à la main, bien décidé à partir en campagne pour tuer le dragon, le roi qui gouverne le pays. Le prince - sous les traits du chancelier Schüssel - se transforme à son tour en dragon comme Jörgi luimême, dès qu'ils ont pris le pouvoir. Dans ce conte destiné aux enfants et à leurs parents, la morale est bien claire: pour être heureux, il faut renoncer au pouvoir et retrouver une véritable âme d'enfant. Sur les derniers dessins, Jörgi rêve de devenir cuisinier devant son chaudron où nage la saucisse, petit élément qui le suit dans tout l'album. Ce qui en fait l'intérêt, c'est la manière dont Haderer dessine les hommes politiques en les sortant de leur contexte habituel.

Dans la représentation de Haider, l'effet de minimisation peut être une manière de tuer le danger que représente la coalition ÖVP-FPÖ en février 2000 en Autriche ${ }^{30}$. Le transformer en enfant peut aussi signifier que le danger potentiel va devenir de plus en plus menaçant. Ainsi, dans l'album, la couronne qu'on lui pose sur la tête est encore trop grande pour lui. Il apparaît comme un petit diable quelque peu maléfique, aux traits stylisés, avec des oreilles de plus en plus pointues. C'est une métaphore souvent en usage dans les partis de la droite autrichienne que de représenter la socialdémocratie sous les traits du diable. Ici, l'allusion est inversée.

Haderer s'en prend également à la mise en scène qui règle les apparitions en public de Haider, à l'éternel sourire qu'on lui voit accroché aux lèvres ${ }^{31}:$ d'agressif sur la page de couverture, où le petit Jörgi se prépare à ses aventures, il devient apaisé à la dernière page. Les angles d'approche varient : la plongée est, bien entendu, destinée à amoindrir la menace, mais les séquences prises en contre-plongée montrent le côté ridicule de ses attitudes; il existe même une vue panoramique quand Jörgi se sent au sommet de sa gloire, dominant la ville du haut du donjon et ne laissant voir que sa chevelure au brushing impeccable.

Dans Jörgi, der Drachentöter, celui qui perce à jour la menace, surveille Jörgi et met en garde les habitants de la ville, c'est le fou du roi. Le message qu'il adresse à tous prend la forme d'un rébus sur un parchemin. Le fou, sorte de clown au nez rouge, coiffé d'un petit chapeau pointu, est une figure récurrente dans les dessins de Haderer pour Stern: c'est toujours lui qui, aux côtés de sa femme, fête la Saint-Sylvestre dans des décors différents, l'air éternellement triste. Ici, il symbolise le bon sens et, grâce à sa vigilance, parvient à déjouer les tours du pouvoir.

35 La dénonciation plus ou moins virulente des adversaires politiques constitue un des éléments favoris de la satire. Haderer l'a pratiquée longtemps, dessinant aussi des 
marionnettes pour la scène du théâtre Im Rabenhof. Son évolution la plus récente est de s'impliquer dans une campagne électorale, celle en faveur du maire social-démocrate de Vienne, Michael Häupl. Comme il nous le confiait au téléphone, s'il s'engage ainsi, c'est pour défendre la démocratie contre les dangers du populisme ${ }^{32}$.

Sous le slogan « Haderer für Häupl », Haderer a lancé l'idée de diffuser des "Fliegende Blätter » (Feuilles volantes). On peut y voir un hommage à la revue satirique munichoise Fliegende Blätter (1884-1940), destinée aux familles ${ }^{33}$. Mais il s'agit, sans doute, davantage de prendre ce terme au sens propre, en tant que supplément du Wiener Bezirksblatt, distribué régulièrement à Vienne ${ }^{34}$. Voici ce qu'en dit Haderer :

Comme la plupart de mes idées, celle des «Fliegende Blätter » est née dans un cercle d'amis, parce que la droite autour de Monsieur Strache s'est à nouveau fait entendre de manière insupportable. J'en avais vraiment marre, et c'est pour cette raison que je veux intervenir en personne dans le combat électoral de Vienne.

Des bandes dessinées vont être distribuées régulièrement dans les foyers viennois, des petits dessins d'ambiances qui distraient, qui irritent ou qui énervent, mais qui en tout cas doivent bien montrer que ce n'est pas une bonne idée de continuer à monter les gens les uns contre les autres avec des phrases creuses $[. . .]^{35}$.

Deux de ces «Fliegende Blätter » étaient déjà parues en juin 2010. Une série de treize était prévue, pouvant être rassemblées dans une pochette, dessinée elle aussi par Haderer. Les planches se présentent sous forme de trois bandeaux comportant deux ou trois vignettes. Pour cesser de "flatter l'ego" des hommes politiques, Haderer y présente des scènes de la vie quotidienne à Vienne. La première se situe dans un café viennois où un client, assis à une table (devant sa proverbiale tasse de café et son verre d'eau posés sur un plateau), lit un journal, y prend, sans doute, connaissance des résultats des élections et réclame au garçon un œillet rouge. Sur la seconde feuille volante, l'action se situe dans une autre salle du même café, car l'on reconnaît à la fois le client et le garçon sur la première vignette. Un club de football donne une conférence de presse pour annoncer que le stade va porter le nom d'une femme : «À la femme de Krankl » (Dem Krankl seiner Frau) tourne en dérision cette nouvelle, rendue comique également par l'usage de la tournure dialectale. La présentation et les thématiques rappellent celles de Moff, la revue de bande dessinée en très petit format et à bas prix que publie Haderer.

Quand il s'attaque au clergé en dévoilant l'immoralisme des moralisateurs, Haderer prône une nouvelle morale qui ne s'embarrasse pas des atermoiements du " politiquement correct ». Sa dénonciation est violente et il clame haut et fort que les puissants, se vautrant sans vergogne dans leur confort et jouissant de leurs privilèges, se comportent très cyniquement en dépit de leurs allégations mensongères. Il prend du recul et de la hauteur par rapport à leur vulgarité. Mais il le fait aussi contre la petite bourgeoisie, une autre de ses cibles favorites, dont il dépeint le ridicule. Il affirme en effet: «Je suis un artiste. Il est important pour moi de poser des questions décisives auxquelles d'autres doivent apporter des réponses ${ }^{36} »$. Et il ajoute encore: "Je n'aimerais pas qu'on attende de chacun de mes travaux une explication du monde. Il s'agit davantage de réfléchir ma perception étonnée de la réalité quand je déplace un peu mon objectif ${ }^{37}$ ".

39 Haderer se définit tout d'abord comme un homme libre, qui choisit ses idées et les défend dans ses dessins en prenant pour cible ses ennemis politiques, situés dans les partis de la droite et de l'extrême droite. Il peut alors cogner jusqu'à l'outrance, sans jamais ménager ses adversaires, ses traits devenant cruels, même quand il s'agit de 
montrer du doigt leurs comparses ou bien ceux qui les suivent aveuglément, sans réfléchir. Il se refuse aussi à être un homme de parti. Son soutien à Häupl et au SPÖ viennois est surtout dirigé contre les déclarations claironnantes des populistes et aux conséquences néfastes que leur victoire électorale entraînerait : «Les ennemis de mes ennemis sont mes amis ", déclare-t-il tout simplement ${ }^{38}$.

Haderer joue un peu lui-même le rôle du fou du roi qui - dans Jörgi, der Drachentöter parvient à ouvrir les yeux aux habitants et, ainsi, à les libérer du pouvoir en place. Certes, dans la réalité, la satire ne peut transformer la société. Mais c'est une arme qui peut contribuer à ouvrir le dialogue, à lutter contre la bêtise et l'abrutissement, l'hypocrisie et le mensonge. En faisant sourire, il aide à vivre mieux, et il le fait avec beaucoup de talent.

\section{NOTES}

1. Nous remercions Gerhard Haderer d'avoir autorisé la publication de quelques dessins dans cet article.

2. Film d'Ulrich Seidl, « Vorsicht Haderer ». Der Cartoonist Gerhard Haderer, co-production ZDF/ ORF, 1992 : « Meine Zeichnungen sind Bildgeschichten. Ich bin ein Erzähler, der eine Geschichte möglichst umfassend zu erzählen hat ».

3. Il a également publié dans Titanic, GEO, Trend, les Oberösterreichische Nachrichten, tenté à la fois par la presse régionale ou nationale et les revues vraiment satiriques.

4. Cela se remarque aussi à son goût pour « l'anti-pub », la contre-publicité, comme dans « United Gags of Benetton », «United Colors of Austria » (deux prêtres, dont l'un déguisé en sœur, avec les traits de Haider, s'embrassent) ou "Reiner Pflanz », où l'on voit deux vendeurs se tordre de rire sous l'affiche qui présente la «bouteille verte », « d'une durée de vie de 3000 ans, provoquant de l'acné et de l'herpès, absolument pas recyclable, et surtout terriblement chère " (in Think positive. Die besten Cartoons aus zehn Jahren, mit einem Vorwort von Reinhard Tramontana und Aufzeichnungen des Künstlers, Wien, Ueberreuter, 1996, sans numéro de page comme dans la plupart des albums de Haderer). Dans ces exemples, Haderer s'en prend à la campagne de publicité de Benetton, mais aussi à la mode de l'écologie, le détournement d'affiches connues contribuant à la subversion.

5. Pour comparaison, le dessinateur Pancho (Le Monde) nous disait exécuter un dessin quotidien en près de trois heures (entretien d'Anne-Marie Corbin avec Pancho le 7 février 2001). Par coquetterie sans doute, Deix, l'un des dessinateurs satiriques autrichiens les plus connus, affirme quant à lui [in Severin Heinisch (éd.), Die Welt des Manfred Deix, Wien, Karikaturmuseum Krems, 2003, pagination absente] à la fois regarder la télévision 18 heures par jour pour y trouver ses idées, lire des piles de journaux, passer des heures dans les cafés, dessiner très vite ou parfois de 15 à 72 heures non-stop. C'est une manière d'éluder la question.

6. «Begnadete Körper» (in Haderer, Think positive. Die besten Cartoons aus zehn Jahren, mit einem Vorwort von Reinhard Tramontana und Aufzeichnungen des Künstlers, Wien, Ueberreuter, 1996, sans numéro de page comme dans la plupart des albums de Haderer). Détournement de l'exercice sur le tapis roulant par la production d'énergie qui actionne la télévision.

7. «Bodybuilder» (in Haderer, Think positive, ibid.). Humour sur les effets d'une interdiction des anabolisants : mise en perspective d'une affiche, représentant un homme aux muscles encore 
proéminents, et de la fonte des muscles généralisée, observée dans la salle d'entraînement; la peau est toute relâchée et fait de savants drapés, noués entre eux pour ne pas gêner les mouvements.

Également in « Vorsicht Haderer!» Karikaturen des Österreichers Gerhard Haderer, Hannover, Verlag Gerd Hatje, 1994, p. 69 : «Anabolika und ihre möglicherweise nicht gänzlich auszuschließenden Nebenwirkungen » (dessin réalisé en 1991).

8. L'une, récemment parue dans Stern (2007, $\left.\mathrm{n}^{\circ} 24\right)$ montre un couple, traversant le désert en voiture décapotable: «Warum trinkt er keine Cola?» («Pourquoi ne boit-il pas de coca?»), confie la femme à son compagnon, en regardant un enfant qui réclame de l'eau. Pour tout commentaire : «Immer mehr Verständnis für die Probleme der Dritten Welt » (« Toujours plus de compréhension pour les problèmes du tiers monde »).

9. Haderer donne deux motifs pour la suppression de sa tribune hebdomadaire ou bi-mensuelle dans Profil : d'une part le relatif manque d'intérêt du public autrichien pour le dessin satirique et la bande dessinée, d'autre part les restrictions budgétaires dues à la crise économique: « Karikatur hat in Österreich leider immer weniger Öffentlichkeit. Mein Herzblatt Profil muss ich aber loben. Die haben 30 Jahre lang Satire auf höchstem Niveau kultiviert, den typischen österreichischen Schmäh eben, dieses Kippen zwischen Tragödie und Satire. Das Aus kam natürlich wegen der Wirtschaftskrise. Das Killer-Argument schlechthin. Wörtlich hieß es : "Profil hat kein Budget mehr für seine wertvollen Cartoonisten." " (Interview de Haderer par Niko Alm, in www.thegap.at)

10. «Wohnlandschaft mit Pferdekopfpflaster aus Oslo » (in Haderer, Think positive, op. cit.).

11. Voir également le film déjà cité d'Ulrich Seidl (note 2), où la méthode de travail de Haderer est décrite avec précision.

12. Haderer, «Zwei ganz normale Tage », in Haderer, Think positive, op. cit. L'hebdomadaire Stern (édition du 17 décembre 1998) en change le titre en «Jetzt wirds gerechter: Auch die Reichen können sich nicht mehr alles leisten » («Il y a maintenant plus de justice : même les riches ne peuvent plus tout s'offrir »).

13. «Ich habe mich als Karikaturist jahrzehntelang für politische Wachsamkeit über den regionalen Tellerrand hinaus eingesetzt und werde das weiter tun ». (Interview in Wiener Bezirksblatt, 31 mai 2010).

14. Reinhard Johler, Herbert Nikitsch et Bernhard Tschofen (éds), Schönes Österreich. Heimatschutz zwischen Ästhetik und Ideologie, Wien, Österreichisches Museum für Volkskunde, 1995. Voir également les actes du Congrès de l'AGES 2007, "Heimat ». La petite patrie dans les pays de langue allemande, Chroniques allemandes, $2009, \mathrm{n}^{\circ} 13$.

15. Sur la définition des clichés et des stéréotypes, voir Joseph Jurt, «Deutsch-französische Fremd- und Selbstbilder in der Literatur und Publizistik der Gegenwart », in Frankreich-Jahrbuch 1995, Opladen, 1995, p.57-79; également Anne-Marie Corbin, L'Image de l'Europe à l'ombre de la Guerre froide. La revue Forum de Friedrich Torberg à Vienne (1954-1961), Paris, L'Harmattan, 2001, p. 19-25.

16. Gerhard Haderer, Von Hunderl und Menschen, Wien, Ueberreuter, 2003. Le titre de l'album est justifié par l'apparition de sept petits chiens, tenus en laisse par une grosse dame. Comme dans les Dix petits nègres d'Agatha Christie, ils disparaissent peu à peu, sur chacune des doubles pages, pour réapparaître miraculeusement à la fin. C'est le seul happy-end, qui contraste avec le chaos final, assez anarchiste, où sombre la ville, dominée par les gangsters, gardes du corps de Mister Oilbush.

17. «Vorsicht, Jäger! Den Brunftschrei des Rothirsches gibts auch als Klingelton fürs Handy!» («Attention, chasseur. Le brame du cerf existe aussi en tant que sonnerie sur le portable !»), in Stern, 2007, $\mathrm{n}^{\circ} 44$.

18. Reinhard Tramontana, «Das Künstlerische », préface de Haderer, in Think positive, op. cit. : «Dies nämlich ist das einzige, was ihn an Prominenten interessiert: das System, das sie 
repräsentieren, ersichtlich $\mathrm{zu}$ machen. Und dieses System als eine Verkettung von Zusammenhängen mit Hilfe grell gemachter Augenblicke, die gern entweder $\mathrm{Zu}$ - oder Un- oder Einzel-Fälle genannt werden, auszuleuchten ».

19. Dans un mail du 16 juin 2010, Haderer nous disait vouloir illustrer ainsi un vieux dicton: «Wie die Nase des Mannes, so sein Johannes ».

20. Gerhard Haderer, Das Leben des Jesus, Wien, Ueberreuter, 2002.

21. L'Ascension du Christ est figurée par deux pieds qui disparaissent tout en haut de la page. Jésus retrouve, d'ailleurs, au Paradis les musiciens et les chanteurs qui plaisent particulièrement à Haderer: John Lennon, Janis Joplin, Bob Marley, Jimmy Hendrix, une harmonie céleste réinterprétée à la mode des années 1970. C'est aussi un clin d'œil du dessinateur à ses lecteurs pour rappeler qu'il a lui-même fondé dans sa jeunesse un groupe de rock qui jouait la musique des Rolling Stones.

22. Haderer introduit toujours des détails amusants : pendant la naissance de Jésus, on ne voit de Marie qu'un pied aux orteils très écartés; mais en même temps, une poule couve ses œufs et douze poussins en sortent et la suivent, élément que l'on retrouve sur chacune des doubles pages, et qui établit une mise en parallèle avec la naissance de Jésus et celle du futur clergé. Traitée ici sur un mode volontairement naïf, cette animalisation des prêtres est un procédé de déconstruction d'un usage fréquent dans le dessin satirique (voir Les Animaux pour le dire. La signification des animaux dans la caricature, Ridiculosa, 2003, $\mathrm{n}^{\circ} 10$ ).

23. L'archevêque Schönborn avait refusé en 1995 qu'une enquête soit menée pour mise en cause du cardinal Groer dans une affaire de pédophilie. Il s'est aussi illustré en 2005 dans le New York Times par ses prises de position contre le darwinisme.

24. «Wer die jahrelangen historischen Recherchen, das penible Studium der Originaltexte, die sündteuren Forschungsreisen und die unzähligen Analysen von Weihrauch, die Gerhard Haderer vorgenommen hat, überprüfen möchte, den verweisen wir auf die diversen Bibel-Ausgaben www.bibel.online.de ou www.biblio.at. »

25. «Der gehört sofort und ersatzlos gestrichen. Das ist ein Relikt aus dem finsteren Mittelalter. Außerdem gibt es ja das Grundrecht auf Freiheit der Kunst, die allerdings oft im Widerspruch dazu steht. Hier herrscht eine völlige Unvereinbarkeit von Aufklärung der letzten 250 Jahre und Kirchendogmen. Das nennt man ja auch den Clash of Civilizations. Mir scheint, als gäbe es derzeit einen Rückzug ins diffus Religiöse. Die schlimmste Ausformung davon ist dieser miserable PolitSlogan "Abendland in Christenhand" » (in www.thegap.at).

26. Interview de Gerhard Haderer dans le Spiegel, 10 février 2006 : « Respektlosigkeit ist die Basis von Karikatur und satirischer Meinungsäußerung, in welcher Form auch immer ».

27. Haderer, in Stern, 27 octobre 1994, «Das unsichtbare Kanzlermodell », in Die ersten zehn Jahre im Stern, Oldenburg, Lappan, 2001, p. 139.

28. Dans une interview, Haderer déclare même apprécier beaucoup Merkel : «Die kann ich um drei Uhr in der Früh im Schlaf zeichnen. Diese Frau setzt alle Vorurteile außer Kraft: Ostdeutsche, Physikerin, Kanzlerin. Sie ist die Antithese zu Helmut Kohl, der ja die typische Karikatur eines Deutschen verkörpert hat. Mächtig, beleibt, aber kleinkariert zugleich. Einmal habe ich Frau Merkel gezeichnet als blauen Engel... ein geradezu idyllisches Blatt ». (in www.thegap.at)

29. Gerhard Haderer, Jörgi, der Drachentöter, Wien, Ueberreuter, 2000. Dans cet album, Haderer utilise toutes les présentations possibles de son histoire en images. Sur des doubles pages, il met en scène la place d'un village à plusieurs moments de la journée et dans le temps et y fait évoluer ses personnages de manière à bien montrer l'évolution de l'ambiance. On trouve aussi des pages simples et d'autres avec plusieurs vignettes. Quant aux éléments textuels, ils résument et commentent l'action qui se déroule, y intégrant entre guillemets les paroles du héros afin de prolonger l'effet comique. Mais Haderer recourt aussi aux bulles. Cette multiplicité de la présentation peut être une manière de ne pas lasser le lecteur, la trame narrative en elle-même 
étant assez faible. Pour les liens entre le texte et l'image, voir Ridiculosa, 1999, n6 (Textuel et visuel).

30. Voir Armin Thurnher (rédacteur en chef de l'hebdomadaire Falter), "Der Feschist ", in Die Zeit, 10 février 2000, n7, p. 3 : «Es kommt ja nicht nur darauf an, was einer sagt. Es kommt auch darauf an, wie einer wirkt. Haiders Geschäft ist das Produzieren von Gesamtbildern, in die sich der provozierende oder abwiegelnde Text, die kalkulierte Provokation samt eingeplantem Dementi oder halbherziger Entschuldigung als organischer Teil einfügt. Den Hintergrund seiner Bilder und Frechheiten gibt jenes alpine Panorama ab, das ich "die Welt des Feschismus" nenne (abgeleitet vom österreichischen Wort "fesch" für stattlich) - und die braucht vor allem einen tüchtigen, anständigen Körper ».

31. Klaus Ottomeyer, Die Haider-Show. Zur Psychopolitik der FPÖ, Klagenfurt, Drava-Verlag, 2000. Voir en particulier les chapitres « Robin Hood in Österreich » et « Der männliche Sportler und die Erotisierung der Politik» (p. 10-51).

32. Entretien téléphonique d'Anne-Marie Corbin avec Haderer le 2 juin 2010.

33. Voir Ursula Koch, « Les Caricaturistes munichois de la Belle Époque face au progrès technique et à ses conséquences ", in Recherches contemporaines, $\mathrm{n}^{\circ}$ spécial L'Image satirique face à l'innovation, Nanterre, PU de Paris X, 1998, p. 65-83.

34. « Das erste dieser "Fliegenden Blätter" wird als Haltestellenplakat affichiert und zusätzlich mittels Feibra verteilt. Insgesamt sind 13 "Fliegende Blätter" geplant, die in einer Sammelmappe - auch von Gerhard Haderer gestaltet - gesammelt werden können. Ab Juni werden diese Karikaturen im Zweiwochen-Abstand erscheinen. Die ersten beiden Ausgaben werden per Feibra verteilt und dem Wiener Bezirksblatt beigelegt. Außerdem liegen sie in den Freecard-Dispensern auf, und man wird sie in der Löwelstraße (Portier) sowie später auch in der Wahlkampfzentrale erhalten.

Das erste der fliegenden Blätter finden Sie weiter unten auch als pdf zum Download. Ab Juni können die Blätter auch auf www.wienerbezirksblatt.at bestellt werden ».

35. « Meine Idee für die "Fliegenden Blätter" ist, wie die meisten meiner Ideen, in einem kleinen Kreis mit ein paar Freunden entstanden, weil die Töne aus der rechten politischen Ecke rund um Herrn Strache wieder einmal besonders unerträglich laut wurden. Ich hab einfach die Nase voll davon und deshalb will ich mich ganz persönlich in den Wiener Wahlkampf einmischen.

So werden ab jetzt regelmässig Cartoons an die Haushalte verteilt, thematisch zwischen Kaisermühlen Blues und Mundl Sackbauer angesiedelt, kleine ironische Stimmungsbilder, die unterhalten, irritieren oder aufregen können, jedenfalls aber klarstellen sollen, dass es keine gute Idee ist, die Menschen mit polemischen Phrasen weiter gegeneinander aufzuhetzen.

Deshalb : Haderer für Häupl. Und mit dem Wiener Bezirksblatt habe ich einen Partner gefunden, dem die Idee auch gefallen hat und so setzen wir das gemeinsam um.» (www.wienerbezirksblatt.at)

36. OÖNachrichten (nachrichten.at), $1^{\mathrm{er}}$ juin 2010 : «Ich bin Künstler. Für mich ist wichtig, entscheidende Fragen zu stellen, die Antworten müssen andere geben ».

37. «Infame Idylle », interview de Gerhard Haderer par Michael Frank (Süddeutsche Zeitung), in Haderer, Danke gut, Wien, Ueberreuter, 2005 : «Ich möchte nicht die Erwartung wecken, dass ich in meinen Arbeiten jeweils die Welt erkläre. Es ist mehr staunendes Reflektieren einer Realität, wie ich sie mit verschobener Optik wahrnehme ».

38. In Falter, 2010, ${ }^{\circ} 22$, p. 17 : « Die Feinde meiner Feinde sind meine Freunde ». 


\section{RÉSUMÉS}

Le dessinateur Gerhard Haderer affirme être un narrateur dont les dessins racontent des histoires en images. Elles prennent diverses formes, depuis le dessin ou la planche dans des hebdomadaires, jusqu'à des albums où l'histoire unique se déroule avec ses péripéties tout au long du volume autour d'un ou de plusieurs héros. Pour présenter les travers de la vie en Autriche, il a mis au point une technique fort élaborée. Son travail, très minutieux, repose sur l'observation des personnes qui l'entourent et du décor dans lequel elles évoluent. Il formule une critique virulente de la société autrichienne et de son provincialisme, rejette l'Église catholique et ses dogmes, met au pilori les hommes politiques de droite et d'extrême-droite. C'est un homme libre, dans la tradition de l'Aufklärung, qui défend ses idées avec talent.

Der Cartoonist Gerhard Haderer behauptet, er sei ein Erzähler, der mit seinen Zeichnungen, Karikaturen und Cartoons Bildgeschichten erzählen wolle, um die Aufmersamkeit seines Publikums auf die unangenehmen und grotesken Seiten des Alltagslebens in Österreich oder auch in Deutschland zu lenken. Dazu hat er eine besonders wirksame Technik erarbeitet. Seine Arbeit beruht auf der Beobachtung der Leute in seiner Umgebung, wie sie in den eigenen vier Wänden, im Büro, in ihrer Stadt leben. Er übt vor allem eine scharfe Kritik an den Zuständen in der österreichischen Provinz, greift die katholische Kirche und ihre Lehre, so wie die rechten und rechtsextremen Politiker an. Haderer ist ein freier Mann, gar ein Aufklärer, der sein Talent unerbittlich in den Dienst seiner Ideen stellt.

Gerhard Haderer is a cartoonist whose drawings are stories told in strings of pictures. They come in various forms, ranging from comic strips and drawings to albums depicting the vicissitudes of one or several main protagonists running through the entire course of one volume. In order to show the failings of life in Austria he has worked out a very sophisticated technique. His minutely detailed work is based on observation of persons close to him and their surroundings. He scathingly criticizes the parochialism of Austrian society, rejecting the Catholic Church and its dogmas and exposing to public scorn politicians of the Right and the extreme Right. He is a free man in the tradition of the Enlightment and applies his talent pitilessly to the defense of his opinions.

\section{AUTEUR}

\section{ANNE-MARIE CORBIN}

Université de Haute-Normandie - Rouen 